\title{
L'Unesco et la bioéthique - de hier à demain
}

\section{Jean Martin}

Membre de la rédaction

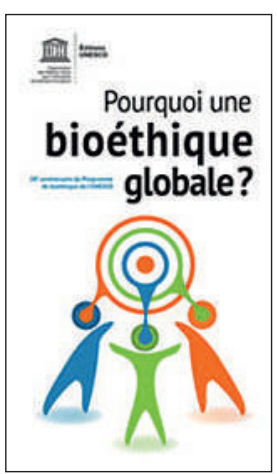

Ouvrage collectif, dirigé par German Solinis Pourquoi une bioéthique globale?

Paris: Editions Unesco; 2015.

168 pages. $29.10 \mathrm{CHF}$. ISBN 978-92-3-200048-4 [1]

1 Cette publication existe aussi en anglais et en espagnol (voir www.unesco.org).

2 Martin J. Le Comité international de bioéthique (CIB) de l'Unesco et la Déclaration universelle sur la bioéthique et les droits de l'homme. Bull Méd Suisses. 2007;88:190-3.
L'Unesco, agence pour l'éducation, la science et la culture, assume depuis les années 1990 le leadership au sein des Nations-Unies du point de vue des préoccupations éthiques multiples dans la société d'aujourd'hui-ceci en collaboration avec d'autres, en particulier l'OMS qui a évidemment sa partie à jouer dans ce domaine. Deux organes jouent un rôle de conseil pour l'Unesco, le Comité international de bioéthique (CIB) [2] et le Comité intergouvernemental de bioéthique (CIGB). Le premier est formé de 36 experts de disciplines diverses (médecine et biologie, philosophie, éducation, droit - et notamment droits humains), s'exprimant de manière indépendante, ad personam, et le second formé de 36 représentants de pays - qui en font partie à tour de rôle. Rappelons qu'une grande réalisation du CIB est l'élaboration de la Déclaration universelle sur la bioéthique et les droits de l'homme (DUBDH), adoptée par la Conférence générale de l'Unesco en octobre 2005 à Tokyo.

D’autres documents majeurs sont la Déclaration universelle sur le génome humain et les droits de l'homme (1997) et la Déclaration internationale sur les données génétiques humaines (2003). Le CIB a aussi rédigé une vingtaine de rapports sur des thèmes liés à la génétique (confidentialité, dépistage, thérapie), sur éthique et neurosciences, sur l'éducation en bioéthique. Ainsi que des documents d'élaboration substantielle d'articles de la DUBDH: ainsi sur le consentement, la responsabilité sociale et la santé, la vulnérabilité humaine, la nondiscrimination et non-stigmatisation.

A l'occasion du $20^{\mathrm{e}}$ anniversaire de son Programme de bioéthique, l'Unesco publie une attrayante plaquette incluant des textes courts de trente auteurs qui ont joué des rôles d'importance en bioéthique, au sein du CIB entre autres. Ces auteurs issus du monde entier incluent Federico Mayor, qui dirigeait l'Unesco lors de la mise en œuvre du Comité, l'actuel et deux ancien(ne)s président(e)s du CIB, deux de ses anciens Secrétaires généraux. Ces contributions sont présentées dans quatre parties: 1) Vision conceptuelle et globale; 2) Aspects philosophiques et normatifs; 3) Expérience, contextualisation et spécificité; 4) Vers l'avenir.

Quelques citations. Sur l'état des lieux: «Des découvertes extraordinaires sur le génome humain ont ouvert des perspectives infinies d'intervention au cours de la vie d'un individu et même au niveau de la conception de cette vie... ouvrant des boîtes de Pandore. En arrière-plan surgissent les vieux fantômes de l'eugénisme et de la libération de Prométhée.»

Sur le but: «Depuis la nuit des temps, l'Homme s'est penché sur le conflit entre ce qui est possible et ce qui est admissible, puisque le savoir est toujours positif, mais son application ne l'est pas toujours [...] La bioéthique cherche à inclure les conséquences du développement technoscientifique dans la société que nous souhaiterions voir advenir, en prenant en compte la question essentielle de la nature humaine, de la conception à la mort [...] L'objectif est double: construire un cadre de référence pour réguler et orienter la gouvernance du savoir technologique et scientifique, et permettre une clarification sociale des alternatives éthiques.»

Sur le mode de travail: «Ce sont les processus de délibération et la construction collective d'opinions, dans le cadre de l'impératif du respect des autres et des différences intrinsèques, qui [en] forment la pierre angulaire, en encourageant un dialogue tripartite entre les experts, les décideurs et les citoyens [...] L’expérience de la négociation sur un pied d'égalité et l'exercice d'un débat pluraliste renforcent la cohésion sociale.»

On pourra trouver ces formulations très générales mais les articles individuels incluent des informations et réflexions pointues. Aussi, nous dit-on: «Le contenu de ce livre n'est délibérément pas homogène, cependant il présente une diversité convergente.» Des impulsions fortes au niveau mondial sont à l'évidence indispensables en matière de bioéthique et il est impératif de tirer profit du cadre planétaire qu'offrent les NationsUnies, en contact avec les Commissions nationales d'éthique et d'autres instances concernées. Impératif de maintenir haut le drapeau des valeurs éthiques et de la réflexion à leur sujet, même si la très inquiétante violence ambiante, internationalement et au sein des pays, met sur le devant de la scène des préoccupations lourdes plus immédiates. 